\title{
Influência da Relação Estequiométrica Resina/Endurecedor em Propriedades Mecânicas de Compósitos Ternários Hierárquicos Resina - Epóxi/Tecido de Fibra de Carbono/Nanotubos de Carbono
}

\author{
Kássio André Lacerda \\ Faculdade de Engenharia e Arquitetura - FEA, Universidade FUMEC \\ Wellington Marcos da Silva, Jefferson José Vilela, Adelina Aparecida dos Santos, \\ Fernando Soares Lameiras, Clascídia Aparecida Furtado \\ Comissão Nacional de Energia Nuclear - CNEN, Centro de Desenvolvimento \\ da Tecnologia Nuclear - CDTN
}

Resumo: A produção de materiais que apresentem alto desempenho em suas aplicações exige avanços tecnológicos e científicos. Os elevados valores de resistência à tração e módulo de elasticidade, aliados à flexibilidade, baixa densidade e alta razão de aspecto, fazem dos nanotubos de carbono excepcionais candidatos para reforçar compósitos poliméricos. Preparamos por laminação sistemas compósitos ternários à base de resina epóxi/tecidos de fibra de carbono (para os binários), acrescendo nanotubos de carbono para os sistemas ternários. Os parâmetros estequiométricos do sistema epoxídico (valor de Phr) e concentração de nanotubos de carbono empregado nos sistemas compósitos tiveram avaliadas suas morfologias (MEV), propriedades térmicas (TG) e mecânicas (ASTM D790). Embora o sistema epoxídico com Phr 10,0 tenha apresentado uma maior estabilidade térmica, os compósitos ternários produzidos a partir do sistema com Phr 26,6 mostraram valores de tensão máxima e módulo de elasticidade até 8 vezes maiores que aqueles produzidos com o sistema Phr 10. A adição de nanotubos de carbono aos compósitos com Phr 26,6 resultou em ganhos adicionais de aproximadamente 38 e $15 \%$ na tensão máxima e no módulo de elasticidade, respectivamente. Esses resultados revelaram a limitação nos métodos de integralização de nanoestruturas a sistemas compósitos, onde as dispersões estão limitadas a uma série de fatores inerentes a interação química e/ou física durante a fabricação dos compósitos nanoestruturados.

Palavras-chave: Resina epóxi, tecidos de fibra de carbono, nanotubos de carbono, relação estequiométrica, propriedades térmicas, propriedades mecânicas.

\section{Influence of Resin/Hardener Stoichiometric Ratios on the Mechanical Properties of Hierarchical Ternary Composites - Epoxy Resin/Woven Carbon Fiber/Carbon Nanotubes}

\begin{abstract}
The production of materials that exhibit high performance in their applications requires scientific and technological advances. The high values of tensile strength and modulus of elasticity, combined with flexibility, low density and high aspect ratio, make carbon nanotubes exceptional candidates for reinforced polymer composites. We prepared by lamination composite systems based on epoxy resin/woven carbon fiber (for binary), plus carbon nanotubes for the ternary systems. The effect of the stoichiometry of the epoxy system (Phr value) and concentration of carbon nanotubes used in the composite systems was evaluated by their morphological (SEM), thermal (TG) and mechanical properties (ASTM D790-10). Although the epoxy system with $10.0 \mathrm{Phr}$ has presented a higher thermal stability, the ternary composite system produced with $26.6 \mathrm{Phr}$ showed values of maximum stress and elastic modulus up to 8 times greater than those produced with the $10 \mathrm{Phr}$ system. The addition of carbon nanotubes to composites with 26.6 Phr resulted in additional increase of approximately 38 and $15 \%$ of the maximum stress and elastic modulus, respectively. These results revealed a limitation in the methods of incorporation of the nanostructures in composite systems, where the dispersion is limited by a number of factors, inherent to the chemical and/or physical interactions during fabrication of nanostructured composites.
\end{abstract}

Keywords: Epoxy resin, carbon fiber woven, carbon nanotubes, stoichiometric relation, thermal properties, mechanical properties. 


\section{Introdução}

É constante a busca por materiais de alto desempenho para os veículos aeroespaciais, cujas metas são a diminuição do índice de massa, o aumento da resistência à fadiga e à corrosão, a redução das emissões e a diminuição dos custos de aquisição e operação, mantendo elevado grau de confiabilidade. A utilização de sistemas compósitos hierárquicos nanoestruturado e o tema central para o avanço tecnológico esperado ${ }^{[1]}$. Compósitos ternários à base de matriz polimérica, nanoestruras e tecido de microfibra como os relatados nesse trabalho poderão ser utilizados no desenvolvimento de satélites e veículos aeroespaciais. Altos valores de condução elétrica e térmica e de resistência à tração e módulo elástico, além de flexibilidade e baixo índice de massa, são propriedades que fazem dos nanotubos de carbono (NT) candidatos a reforçar materiais compósitos de matrizes poliméricas para setores estratégicos ${ }^{[2-7]}$. Contudo, aliar as propriedades mecânicas e condutoras dos reforços (fibra de carbono e NT) às características de baixa densidade, facilidade de processamento, estabilidade dimensional e baixa toxicidade dos sistemas epóxi é um desafio que requer principalmente ${ }^{[8]}:$ 1) dispersão e adesão dos NT à resina ${ }^{[9]}$; 2) alto grau de molhamento do tecido de fibra de carbono com a dispersão epóxi/NT; e 3) otimização das condições de processamento do sistema polimérico na presença das cargas. Com a descoberta em 1991 dos $\mathrm{NT}^{[2]}$, intensificou a busca da integralização dessas estruturas a materiais com grande solicitações mecânicas e condutoras. Mas há muito, os trabalhos revelaram as dificuldades desse processo ${ }^{[10]}$. Várias rotas de processamento de natureza química e física foram tentadas, mas com resultados limitados na distribuição e interação das nanoestruturas com os meios de interesse em aplica-los. Muito desses resultados estão atribuídos a variabilidade dessa nanoestrutura, em termos de composição química das amostra de NT, tipos de nanotubos (parede simples/múltiplas; quiralidade [zig-zag, quiral, armchair], extremidades abertas ou fechadas), parâmetros geométricos (diâmetro, comprimento dos tubos) e reatividade química dos vários tipos de nanotubos de carbono, estado de agregação (tubos isolados ou em feixes) ${ }^{[5,11]}$. Processamentos químicos como dispersão em meios ácidos, solventes orgânicos, soluções com moléculas surfactantes e macromoléculas foram tentados pautados pelas interações intermoleculares com os nanotubos. Outras alternativas foram as funcionalizações com caráter covalente da superfície e extremidade dos nanotubos, essa via melhora a interação dos mesmos com as matrizes e rompe a conexão entre os vários tubos que tende a forma feixe durante os processos de síntese ${ }^{[12,13]}$. Outras estratégias de natureza física foram tentadas com a moagem em moinhos de bolas, calandragem de nanotubos em polímeros e a sonicação vigorosa de nanotubos em solventes diversos e polímeros viscosos ${ }^{[11]}$.

Neste contexto, investigamos a influência da estequiometria da reação de cura dos compósitos binários e ternários baseados em resina epóxi éter de diglicidil bisfenol A, endurecedor 4'4-diaminodifenilmetano, tecido 2D de fibra de carbono e nanonanotubos de carbono de paredes múltiplas (MWNTs, do inglês multi walled carbon nanotubes) nas suas propriedades térmicas e mecânicas (tensão máxima e módulo de elasticidade). Sistemas binários e ternários com variações no parâmetro estequiométrico $\mathrm{Phr}$ e em percentuais mássicos de MWNT foram preparados, ao final foram caracterizados por microscopia eletrônica de varredura (MEV), termogravimetria (TG), calorimetria exploratória diferencial (DSC, do inglês differencial scanning calorimetry) e ensaios de tração ${ }^{[14]}$.

\section{Materiais e Métodos}

A resina epóxi éter de diglicidil bisfenol A (DGEBA), denominada ARALDITE ${ }^{\circledR}$ MY 750, foi a matriz escolhida. Esta resina é líquida, de baixa viscosidade (a $25^{\circ} \mathrm{C}-12.000-16.000 \mathrm{mPa} / \mathrm{s}$ ) e densidade de $1,16 \mathrm{~g} / \mathrm{cm}^{3}$. $\mathrm{O}$ agente de cura ou "endurecedor", referenciado como Aradur ${ }^{\circledR}$ HT 972 GB, é uma diamina aromática sólida denominada 4'4-diaminodifenilmetano (DDM), com densidade de $0,98 \mathrm{~g} / \mathrm{cm}^{3}$ e temperatura de transição vítrea entre $153-170{ }^{\circ} \mathrm{C}$. Ambos, matriz e agente de cura, foram fabricados pela empresa Huntsman ${ }^{\circledR}$.

O tecido de fibra de carbon (TFC) do tipo "plain" foi fabricado pela empresa Hexcel Corporation ${ }^{\circledR}$ com fibras de carbono HexTow AS4C. As fibras AS4C são fibras aeronáuticas produzidas a partir de poliacrilonitrila (PAN) com 3000 filamentos por cabo. Estas fibras recebem tratamento com resina epóxi em sua superfície para melhorar a interação matriz-fibra e apresentam alta resistência à tração $(4,4 \mathrm{GPa})$ e alto módulo elástico (231 GPa).

$\mathrm{O}$ reforço nanoestruturado utilizado foi o MWNT denominado Ctube 100, produzido pela empresa NC CO. LTD. De acordo com o fabricante, as amostras de MWNT apresentam alto grau de pureza ( $>93 \%$ em massa), diâmetro médio de tubos de 10 a $50 \mathrm{~nm}$, comprimento entre 1 e $25 \mu \mathrm{m}$, densidade de 0,03-0,06 g/ $\mathrm{cm}^{3}$ e área superficial específica de 150 a $250 \mathrm{~m}^{2} / \mathrm{g}^{[15]}$.

\section{Obtenção dos sistemas compósitos}

Uma mistura resina epóxi/MWNT foi inicialmente agitada por $30 \mathrm{~min}$ a $2400 \mathrm{rpm}$ com agitador mecânico e, em seguida, sonificada por inserção de uma ponta sonificadora (stepped microtip S\&M 219, ф 13,0 mm), à potência média de $30 \mathrm{~W}$ e amplitude de $35 \%$, por 60 min, utilizando-se o equipamento Sonics VibraCell VCX500. Sob aquecimento $\left(60{ }^{\circ} \mathrm{C}\right)$ e agitação mecânica (120 rpm), adicionou-se o agente de cura até sua completa solubilização na mistura resina/MWNT. Foram preparadas concentrações de $0,1,0,5$ e 1,0\% em massa de MWNT.

A relação estequiométrica entre resina epóxi e endurecedor é representada pelo parâmetro "partes em massa de agente de cura por cem partes de resina epóxi" (Phr, do inglês parts of amines per hundred of resins):

$$
P h r_{A \min a}=\frac{\left[\frac{M-N H_{3}}{n^{\mathrm{o}} H}\right] \times 100}{\frac{\overline{M n}}{F}}
$$


tem-se no numerador o MEHA, a massa equivalente de hidrogênios ativos, e no denominador o MEE, a massa equivalente da molécula epóxi (DGEBA). Os parâmetros da equação são: $M-\mathrm{NH}_{3}$ - massa molar do grupamento amina; $n^{o} H$ - número de hidrogênios ativos; $\overline{M n}$ - massa molar média da molécula epóxi (DGEBA); $F$ - funcionalidade (número médio de sítios epóxi reativos por monômero do polímero DGEBA).

Os nanocompósitos foram obtidos por um processo de laminação de camadas do sistema epoxídico com Phr 10,0 e 26,6, seguindo o arranjo de laminação [0] (camada $_{4}$ de resina/MWNT, tecido de fibra de carbono, camada de resina/MWNT e assim sucessivamente, até o enchimento do molde).

O procedimento de cura iniciou-se com uma razão de aquecimento de $6{ }^{\circ} \mathrm{C} / \mathrm{h}$, da temperatura ambiente até $60{ }^{\circ} \mathrm{C}$, permanecendo nesta temperatura por $1 \mathrm{~h}$; com a mesma razão de aquecimento, o sistema foi aquecido até atingir $120^{\circ} \mathrm{C}$, permanecendo nesta temperatura por $2 \mathrm{~h}$; e, em seguida, até $180{ }^{\circ} \mathrm{C}$, ficando por $1 \mathrm{~h}$ nesta temperatura. $\mathrm{O}$ sistema foi resfriado a uma razão de $20{ }^{\circ} \mathrm{C} / \mathrm{h}$ até $30^{\circ} \mathrm{C}$.

\section{Caracterização}

Amostras de aproximadamente $15,0 \mathrm{mg}$ foram analisadas por TG em um equipamento TA Instruments modelo SDT 2960. O protocolo aplicado para as medidas foi razão de aquecimento de $5{ }^{\circ} \mathrm{C} / \mathrm{min}$, da temperatura ambiente até $1000{ }^{\circ} \mathrm{C}$, sob atmosfera de ar sintético seco ou nitrogênio, com fluxo de $100 \mathrm{~mL} / \mathrm{min}$. As medidas de DSC foram realizadas em um equipamento DSC Q10 da TA Instruments. As amostras foram analisadas conforme protocolo estabelecido na norma técnica ASTM D 3418-08 ${ }^{[17]}$.

As micrografias eletrônicas de varredura foram obtidas em um equipamento JEOL JSM, modelo 840A, operando com o feixe de elétrons sob tensão de aceleração de $15 \mathrm{kV}$, corrente de $6 \times 10^{-11} \mathrm{~A}$. Os compósitos fraturados durante os ensaios mecânicos foram mergulhados em nitrogênio líquido e depois rompidos na região de fratura. As amostras dos compósitos foram metalizadas com ouro por $10 \mathrm{~s}$ na região da fratura e analisadas.

Os ensaios de flexão para os nanocompósitos ternários seguiram a norma técnica ASTM D790M-10 $0^{[14]}$, método de ensaio tipo I (procedimento A). Para tais ensaios foi utilizada uma máquina universal de ensaios mecânicos EMIC, modelo DL3000.

\section{Resultado e Discussão}

A relação estequiométrica entre resina (DGEBA) e agente de cura (DDM) define o grau de reticulação do sistema epoxídico e influencia, portanto, a qualidade da dispersão e adesão de cargas a este sistema e as propriedades térmicas e mecânicas de sistemas epoxídicos puros e compósitos ${ }^{[18,19]}$. Esta relação estequiométrica foi representada pelo parâmetro Phr. Os valores $\mathrm{Phr}$ 10,0 e 26,6 foram escolhidos para avaliação: este último, usualmente escolhido nas formulações convencionais, por corresponder à relação molar de $1 \mathrm{~mol}$ de DDM/2 moles de DGEBA, supondo que todos os hidrogênios ativos do grupo amina sejam substituídos pelos grupos epóxi na reação de cura; e Phr 10,0 por corresponder à relação $1 \mathrm{~mol}$ de DDM/5 moles de DGEBA, que, embora represente um grau de reticulação menor, poderia acarretar numa maior fluidez do sistema, favorecendo a dispersão dos NT e a molhabilidade de ambos os reforços NT e fibra de carbono. A Tabela 1 apresenta as relações estequiométricas DDM/DGEBA Phr 10,0 e 26,6.

A Figura 1 apresenta as curvas TG em atmosfera de ar sintético seco para os sistemas epoxídicos após o procedimento de cura. Os percentuais de perda de massa e as temperaturas de decomposição são apresentados na Tabela 2. Os valores de temperatura de transição

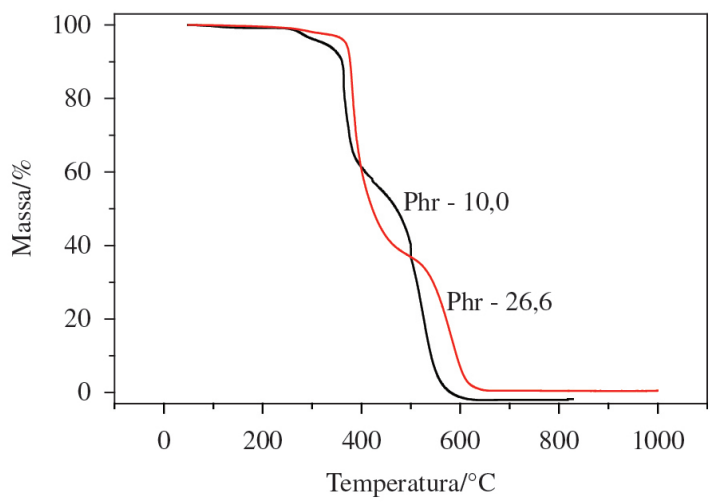

Figura 1. Curvas TG em atmosfera de ar sintético seco para sistemas epoxídicos com Phr 10,0 e 26,6; razão de aquecimento de $5{ }^{\circ} \mathrm{C} / \mathrm{min}$.

Tabela 1. Relações estequiométricas entre resina epóxi e agente de cura.

\begin{tabular}{cccccc}
\hline $\mathbf{P h r}$ & $\mathbf{N}^{\mathbf{0}}$ Mol DDM & $\mathbf{N}^{\mathbf{0}}$ Mol DGEBA & $\boldsymbol{N}^{\mathbf{1}}$ & \% DDM & \% DGEBA \\
\hline 10,0 & 0,0050 & 0,0268 & 0,19 & 9,1 & 90,9 \\
26,6 & 0,0134 & 0,0268 & 0,50 & 21,0 & 79,0 \\
\hline
\end{tabular}

${ }^{1} \mathrm{~N}=\mathrm{n}^{\circ} \mathrm{mol} \mathrm{DDM} / \mathrm{n}^{\circ} \mathrm{mol}$ DGEBA.

Tabela 2. Perdas de massa e temperaturas de decomposição térmica em atmosfera de ar seco para os sistemas epoxídicos com Phr 10,0 e 26,6 .

\begin{tabular}{ccccccc}
\hline Sistema epoxídico & \multicolumn{2}{c}{$\mathbf{1}^{\mathbf{a}}$ Perda de massa } & \multicolumn{2}{c}{$\mathbf{2}^{\mathbf{a}}$ Perda de massa } & \multicolumn{2}{c}{$\mathbf{3}^{\text {a Perda de massa }}$} \\
\cline { 2 - 7 } & $\mathbf{\%}$ & \multicolumn{1}{c}{$\mathbf{C}$} & $\boldsymbol{\%}$ & ${ }^{\circ} \mathbf{C}$ & $\boldsymbol{\%}$ & ${ }^{\circ} \mathbf{C}$ \\
\hline Phr 10,0 & 4,5 & $100-320^{1}(282)^{2}$ & 38 & $320-460^{1}(365)^{2}$ & 57 & $460-640^{1}(534)^{2}$ \\
Phr 26,6 & 2,0 & $100-320^{1}(289)^{2}$ & 60 & $320-500^{1}(382)^{2}$ & 38 & $500-680^{1}(584)^{2}$ \\
\hline
\end{tabular}

${ }^{1}$ intervalo de decomposição térmica; ${ }^{2}$ picos de decomposição térmica. 
vítrea encontrados para as resinas epóxi reticuladas com Phr 10,0 e 26,6 foram 167 e $172{ }^{\circ} \mathrm{C}$, respectivamente. Esses valores foram determinados tomando o ponto de inflexão da curva DSC para essas amostras e estão bem próximos dos valores encontrados na literatura para outros sistemas epoxídicos ${ }^{[20]}$. O maior valor $\mathrm{Tg}$ para o sistema com Phr 26,6 reflete o seu maior grau de reticulação. $\mathrm{O}$ aumento da densidade de reticulação reduz a mobilidade da cadeia, aumentando, portanto, a $\mathrm{Tg}^{[21-23]}$. Ambos os sistemas Phr 10,0 e 26,6 revelaram três estágios de degradação térmica. A primeira perda de massa, em torno de 4,5\% para $\mathrm{Phr} 10,0$ e 2,0\% para Phr 26,6, ocorreu entre 100 e $320^{\circ} \mathrm{C}$, com os respectivos picos de decomposição em $282{ }^{\circ} \mathrm{C}$ e $289^{\circ} \mathrm{C}$. Esta perda inicial é atribuída à volatilização de segmentos de baixa massa molar que não se reticularam durante o processo de cura ${ }^{[24]}$. A segunda perda de massa ocorreu entre 320 e $500{ }^{\circ} \mathrm{C}$, com picos de decomposição em $365^{\circ} \mathrm{C}$ e $382^{\circ} \mathrm{C}$ para $\mathrm{Phr} 10,0$ e 26,6 , respectivamente. A terceira perda de massa ocorreu entre 460 e $680{ }^{\circ} \mathrm{C}$, estando os picos de decomposição em valores agora bastante distintos, $534{ }^{\circ} \mathrm{C}$ para $\mathrm{Phr} 10,0$ e $584{ }^{\circ} \mathrm{C}$ para $\mathrm{Phr} 26,6$. Estes dois últimos estágios de perda de massa são atribuídos à decomposição de segmentos de alta massa molar, formados a partir da reticulação da resina epóxi ${ }^{[24]}$. Embora o percentual em massa do retículo mais estável termicamente seja maior para o sistema epoxídico $\mathrm{Phr}$ $10,0(57 \%)$, a menor quantidade relativa de endurecedor nesse sistema resultou, entretanto, em um percentual de aproximadamente $4,5 \%$ em massa de material não reticulado e na diminuição de até $50{ }^{\circ} \mathrm{C}$, em relação ao sistema com Phr 26,6, da estabilidade térmica dos segmentos dos retículo mais estáveis.

Os valores de temperatura de transição vítrea encontrados para as resinas epóxi reticuladas com $\mathrm{Phr}$ 10,0 e 26,6 foram 167 e $172{ }^{\circ} \mathrm{C}$, respectivamente. Esses valores foram determinados tomando o ponto de inflexão da curva DSC para essas amostras e estão bem próximos dos valores encontrados na literatura para outros sistemas epoxídicos $^{[20]}$. O maior valor de $\mathrm{Tg}$ para o sistema com Phr 26,6 reflete o seu maior grau de reticulação. O aumento da densidade de reticulação reduz a mobilidade

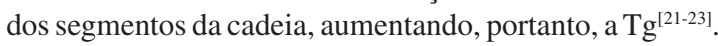

\section{Avaliação das propriedades mecânicas dos compósitos ternários sistema epóxi/fibra de carbono/MWNT}

Os ensaios mecânicos de dobramento em três pontos nos sistemas epoxídios com Phr 10,0 e 26,6 apresentaram comportamentos distintos: o sistema com Phr 10,0 mostrou um comportamento elástico característico dos materiais elastômeros. O ensaio chegou ao fim sem o rompimento do corpo de prova, mostrando que a baixa concentração de endurecedor diminuiu o grau de reticulação da rede termorrígida. Para o sistema estequiométrico ( $\mathrm{Phr} 26,6$ ), o regime elástico se estendeu até a fratura sequenciada e os valores de tensão máxima e módulo de elasticidade aumentaram em até 8 vezes (Tabela 3). Como esperado, os índices mecânicos alcançados nessa avaliação para o sistema binário resina epóxi/fibra de carbono foram menores que aqueles apresentados para compósitos clássicos de resina epóxi e tecidos 2D de fibras de carbono fabricados pela indústria aeroespacial, uma vez que aqui trabalhamos com um percentual em volume de tecido de fibra bem menor $(23-33 \%)^{[10]}$. Para os compósitos ternários com Phr 10,0, a adição de MWNT resultou em valores ainda menores de tensão máxima, em relação ao compósito binário, e não alterou de forma significativa os valores de módulo de elasticidade. Os melhores valores foram encontrados para o compósito contendo 0,5\% em massa de MWNT. Já em relação aos compósitos ternários com $\mathrm{Phr}$ 26,6, a composição de $0,5 \%$ em massa de MWNT apresentou diminuição tanto da tensão máxima quanto do módulo de elasticidade. Ganhos em propriedades foram obtidos, entretanto, para as composições de $0,1 \%$ e, principalmente, $1,0 \%$ em massa de MWNT(respectivamente 38 e $15 \%$ de aumento). Ganhos não forma significativos com a introdução de componentes com propriedades mecânicas consideradas excepcionais (1,0 TPa de módulo elástico para o MWNT). Os resultados mecânicos refletem a heterogeneidade dos compósitos obtidos, tanto no que se refere às dimensões dos corpos de prova, quanto à dispersão de MWNT e interação efetiva dos componentes do compósito. Esta heterogeneidade foi ocasionada principalmente pela limitação nos procedimentos laboratoriais de dispersão/adesão dos reforços e laminação dos compósitos. Para conseguir alcançar as melhorias

Tabela 3. Avaliação das propriedades mecânicas dos compósitos ternários com estequiometrias Phr 10,0 e 26,6 ASTM D790M ${ }^{[14]}$.

\begin{tabular}{|c|c|c|c|c|}
\hline \multicolumn{5}{|c|}{ Sistemas compósitos com Phr 10,0} \\
\hline \multirow{2}{*}{ Sistemas } & \multicolumn{2}{|c|}{ Volume Reforço (\%) } & \multirow{2}{*}{ Tensão Máxima (MPa) } & \multirow{2}{*}{$\begin{array}{c}\text { Módulo de } \\
\text { elasticidade(GPa) }\end{array}$} \\
\hline & TFC & MWNT & & \\
\hline B-MWNT0,0\% & $26,89 \pm 1,58$ & $0,0 \pm 0,0$ & $76,17 \pm 11,24$ & $4,10 \pm 1,12$ \\
\hline T-MWNT0,1\% & $23,88 \pm 0,42$ & $2,07 \pm 0,01$ & $54,44 \pm 4,78$ & $3,27 \pm 0,65$ \\
\hline T-MWNT0,5\% & $32,01 \pm 1,42$ & $10,12 \pm 0,52$ & $59,70 \pm 5,82$ & $5,98 \pm 1,31$ \\
\hline T-MWNT1,0\% & $29,93 \pm 2,14$ & $19,98 \pm 0,48$ & $50,51 \pm 3,94$ & $4,15 \pm 2,28$ \\
\hline \multicolumn{5}{|c|}{ Sistemas compósitos com Phr 26,6 } \\
\hline \multirow{2}{*}{ Sistemas } & \multicolumn{2}{|c|}{ Volume Reforço (\%) } & \multirow{2}{*}{ Tensão Máxima (MPa) } & \multirow{2}{*}{$\begin{array}{c}\text { Módulo de } \\
\text { elasticidade(GPa) }\end{array}$} \\
\hline & TFC & MWNT & & \\
\hline B-MWNT0,0\% & $25,01 \pm 0,82$ & $0,0 \pm 0,0$ & $291,00 \pm 47,27$ & $17,13 \pm 2,62$ \\
\hline T-MWNT0,1\% & $26,40 \pm 2,08$ & $1,97 \pm 0,03$ & $299,43 \pm 23,83$ & $17,96 \pm 1,16$ \\
\hline T-MWNT0,5\% & $25,32 \pm 0,85$ & $9,45 \pm 0,05$ & $285,97 \pm 31,53$ & $14,59 \pm 1,45$ \\
\hline T-MWNT1,0\% & $25,94 \pm 0,79$ & $19,59 \pm 0,38$ & $403,02 \pm 32,31$ & $19,71 \pm 2,64$ \\
\hline
\end{tabular}


pretendidas; aumentos nos percentuais volumétricos de elementos reforços (atingindo 65\% do volume do corpo de prova com tecidos fibrosos e MNWT), a redução das variações dimensionais devido a sistemas automatizados de controle dos parâmetros temperatura e pressão durante o processamento dos compósitos, maior adesão e maior dispersão/adesão entre respectivamente os tecidos fibrosos e os MWNT à matriz polimérica obtidas por modificação das superfícies dos reforços são direcionamentos claros, mas muitos deles ainda desafios atuais.

Imagens de MEV na Figura 2 ilustram a heterogeneidade dos sistemas. Em geral, resinas epoxídicas apresentam fraturas frágeis, de aparência lisa, com linhas contínuas de propagação de trincas ${ }^{[25]}$.
$\mathrm{Na}$ Figura 2, a clivagem unidirecional está presente nas duas estequiometrias ( $\mathrm{Phr}$ 10,0 e 26,6) do sistema epoxídico (Figures 2a e b). Entretanto, a resina com Phr 10 (Figure 2a) apresentou comportamento clássico de superfície de fratura lisa e espelhada. A resina com Phr 26,6 (Figure 2b) apresentou uma morfologia estriada com alta rugosidade. A inclusão de MWNT não alterou a superfície de fratura, a clivagem unidirecional está presente em todas as formulações $0,0 \%, 0,1 \%, 0,5 \%$ e 1,0\% em massa de MWNT. No entanto, a dispersão não é homogênea para esses sistemas. A Figura 2c mostra regiões com bolsões de MWNTs aglomerados (destacados por um círculo). A ampliação das imagens da fratura sobre uma região de aglomerados (Figure 2d)

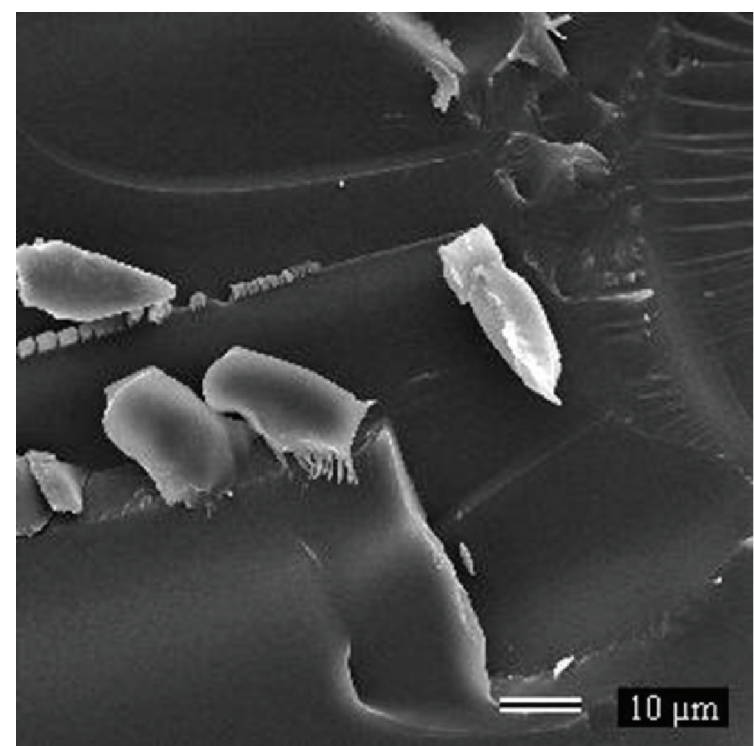

(a) Resina Phr 10

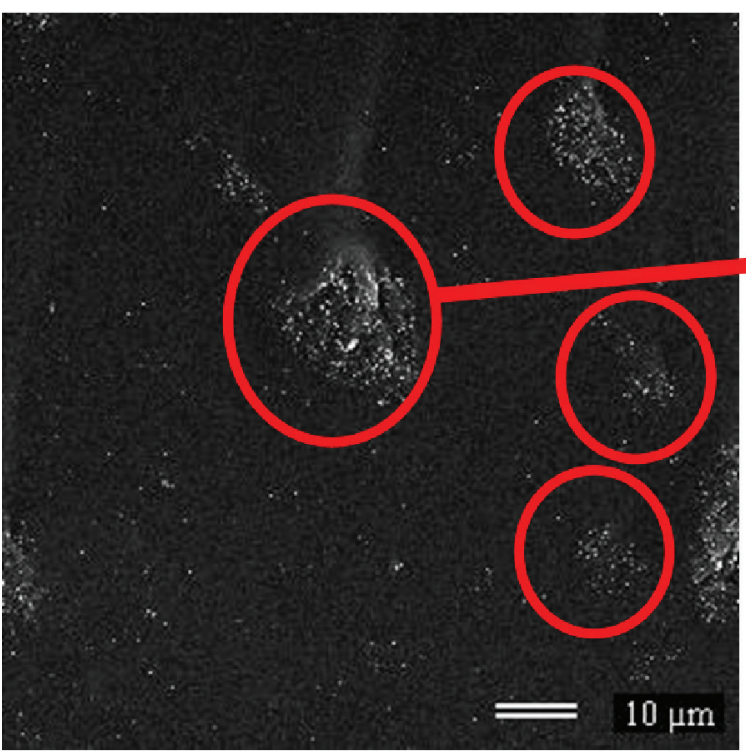

(c) Resina Phr 26,6 com 1\% m/m de MWNT

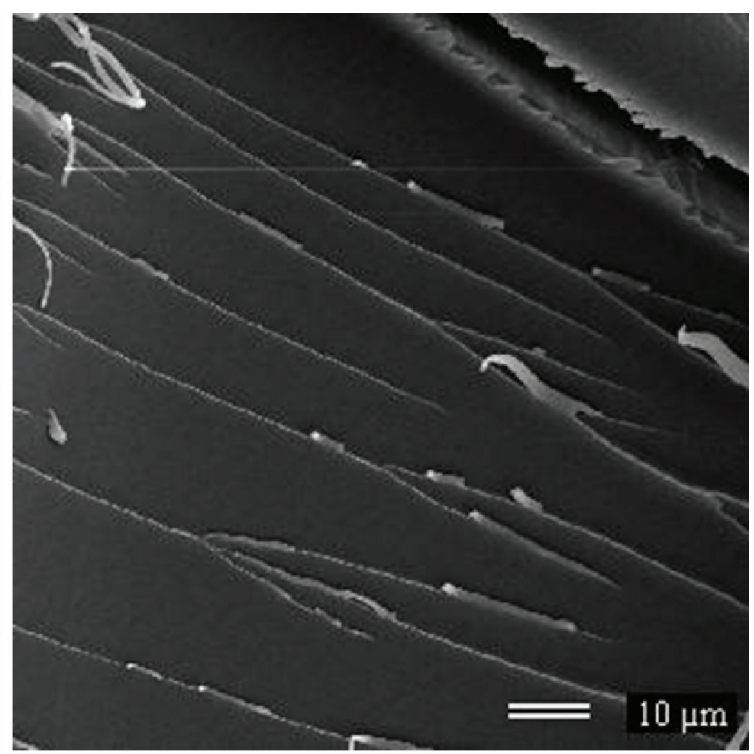

(b) Resina Phr 26,6

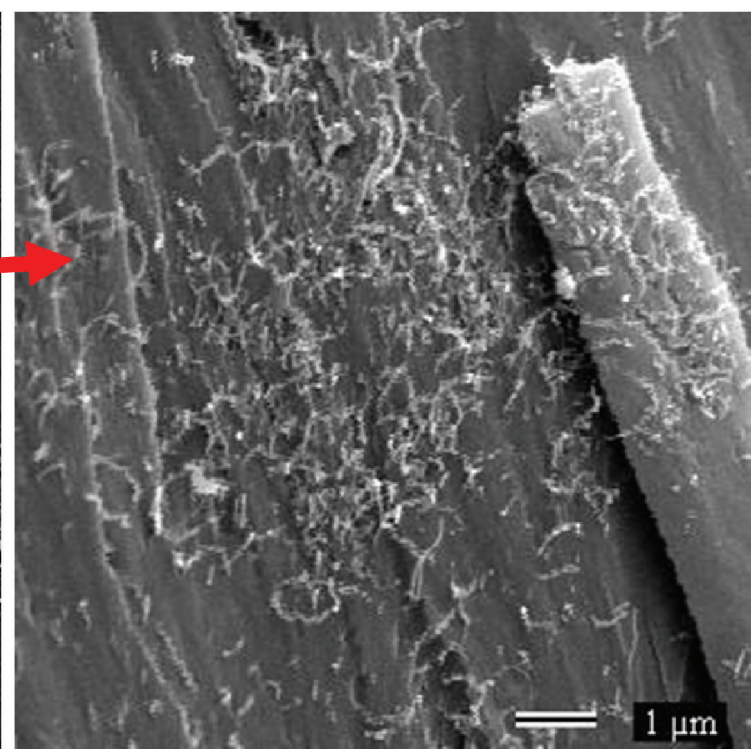

(d) Resina Phr 26,6 com 1\% m/m de MWNT

Figura 2. Imagens MEV para sistemas epoxídicos à base de DGEBA (MY 750) e DDM (HT 972), em alta ampliação e variando a formulação de cura: (a) Phr 10,0; (b) Phr 26,6; (c) e (d) compósitos binários resina epóxi (Phr 26,6)/MWNT. 
revela, porém, uma distribuição uniforme dos MWNTs nessa região, principalmente para o sistema com maior volume de carga de MWNT.

\section{Conclusões}

Compósitos ternários resina epóxi/fibra de carbono/ MWNT foram confeccionados para duas relações estequiométricas resina/endurecedor diferentes, a saber, Phr 10,0 e 26,6. Para ambos os sistemas reconhece-se a limitação na dispersão de nanoestruturas. A falta de uniformidade na dispersão afetou forma significante os índices mecânicos, contudo ganhos foram visto com inclusão de MWNT nas duas condições testadas (Phr 10,0 e 26,6). Para o sistema de Phr 10,0 os compósito com $0,5 \%$ de MWNT (m/m) mostrou-se melhor, enquanto, que no sistema $\mathrm{Phr} 26,6$ os ganhos forma mais pronunciados nos percentuais 0,1 e 1,0\% de MWNT $(\mathrm{m} / \mathrm{m})$. Esse fatos indicam que os processos de dispersão deve ser melhorados e a busca por novos métodos serão necessários. O sistema epoxídico $\mathrm{Phr}$ 26,6 apresentou maior grau de reticulação, maior estabilidade térmica dos segmentos reticulados e maior temperatura de transição vítrea. Os compósitos obtidos a partir desse sistema epoxídico ( $\mathrm{Phr}$ 26,6) apresentaram valores superiores de tensão máxima e módulo de elasticidade. A presença de MWNT em até 1,0\% em massa nesses últimos compósitos aumentou os valores de tensão máxima em até $38 \%$ e do módulo de elasticidade em até $15 \%$. A heterogeneidade nos valores obtidos foram, entretanto, significativa e reflete a baixa dispersão e adesão dos MWNTs na matriz epóxi. A aglomeração dos nanoreforços em regiões colabora para a formação de núcleos de tensão, fazendo com que os incrementos nos módulos mecânicos sejam ainda modestos.

\section{Agradecimentos}

CDTN/CNEN, REDEMAT (UFOP/CETEC/ UEMG), CNPq, CAPES, FAPEMIG, AEB, Rede Nacional de Pesquisa em Nanotubos de Carbono/ CNPq, Instituto Nacional de C\&T de Nanomateriais de Carbono/CNPq.

\section{Referências Bibliográficas}

1. Quian, H.; Greenhalgh, E. S.; Shaffer, M. S. P. \& Bismarck, A. - J. Mater. Chem., 20, p.4751 ( 2010). http://dx.doi. org/10.1039/C000041H

2. Iijima, S. - Nature, 354, p.56(1991). http://dx.doi. org/10.1038/354056a0

3. Njuguna, J. \& Pielichowski, K. - Adv. Eng. Mater., 6, p.193 (2004). http://dx.doi.org/10.1002/adem.200305111

4. Njuguna, J. \& Pielichowski, K. - Adv. Eng. Mater., 6, p.204 (2004). http://dx.doi.org/10.1002/adem.200305110

5. Zhu, J.; Kim, J. D.; Peng, H.; Margrave, J. L.; Khabashesku, V. N. \& Barrera, E. V. - Nano Lett., 3, p.1107 (2003). http:// dx.doi.org/10.1021/n10342489
6. Coleman, J. N.; Khan, U., Blau, W. J. \& Gun'ko, Y. K. - Carbon, 44, p.1624 (2006). http://dx.doi.org/10.1016/j. carbon.2006.02.038

7. Giménez, R.; Fernandez-Francos, X.; Salla, J. M.; Serra, A.; Mantecon, A. \& Ramis, X. - Polymer, 46, 10637 (2005). http://dx.doi.org/10.1016/j.polymer.2005.09.026

8. Costa, M. L.; Pardini, L. C. \& Rezende, M. C. - Mater. Res., 8, p.65(2005).

9. Santos, A. S.; Leite, T. O. N.; Furtado, C. A.; Welter, C.; Pardini, L. C. \& Silva, G. G.- J. Appl. Polym. Sci., 108, p.979 (2008). http://dx.doi.org/10.1002/app.27614

10. Du, J. H.; Bai, J. \& Cheng, H. M. - Expr. Polym. Lett., 1, p.253 (2007). http://dx.doi.org/10.3144/expresspolymlett.2007.39

11. Chen, W.; Auad, M. L.; Williams, R. J. J. \& Nutt, S. R. - Eur. Poly. J., 46, p.2765, (2006). http://dx.doi.org/10.1016/j. eurpolymj.2006.04.016

12. Wang, S.; Liang, Z.; Liu, T.; Wang, B. \& Zhang, C. - Nanotechnology, 17, p.1551, (2006). http://dx.doi. org/10.1088/0957-4484/17/6/003

13. Gojny, F. \& Schulte, K. - Compos. Sci. Techonol., 64, p.2303, (2004). http://dx.doi.org/10.1016/j.compscitech.2004.01.024

14. American Society For Testing And Materials. - "ASTM-D-790-10", in: Annual book of ASTM standards, p.1, ASTM, Philadelphia (2010).

15. CNT Co. Ltd. - "Catalogue". Disponível em: <http://www. carbonnanotube.biz/catalogue.pdf $>$. Acessado em: 29 abr. 2008

16. May, C. A. \& Tanaka, Y. - Epoxy Resins: Chemistry and Technology, Marcel Dekker, INC., New York, p.801, (1973).

17. American Society For Testing And Materials. - "ASTM-D-3418-08", in: Annual book of ASTM standards, p.1, ASTM, Philadelphia (2008).

18. Francis, B.; Thomas, S.; Thomas, S. P.; Ramaswamy, R. \& Lakshmana Rao, V. - Colloid Polym. Sci., 285, p.83 (2006). http://dx.doi.org/10.1007/s00396-006-1537-0

19. Pires, G.; et al. - Rev. Mater., 10, p.317 (2005).

20. Tarifa, S. \& Bouazizi, A. - J. Therm. Anal., 48, p.297 (1997). http://dx.doi.org/10.1007/BF01979273

21. Moon, C. R.; Bang, B. R.; Choi, W. J.; Kang, G. H. \& Park, S. Y. - Polym. Test., 24, p.376 (2005). http://dx.doi. org/10.1016/j.polymertesting.2004.10.002

22. Parra, D. F.; Mercuri, L. P.; Matos, J. R.; Brito, H. F. \& Romano, R. R. - Thermochim. Acta, 386, p.143 (2002). http://dx.doi.org/10.1016/S0040-6031(01)00809-7

23. Nieu, N. H.; Tan, T. T. M. \& Huong, N. L. - J. Appl. Polym. Sci., 61, p.2259 (1996). http://dx.doi.org/10.1002/ (SICI) 1097-4628(19960926)61:13<2259::AIDAPP3>3.0.CO;2-B

24. Loss, M. R.; Coelho, A. F. \& Pezzin, S. - Polímeros, 18, p.76 (2008).

25. Romão, B. M. V. - Polímeros, 13, p.173 (2003). http:// dx.doi.org/10.1590/S0104-14282003000300007

Enviado: 08/03/13 Reenviado: 01/07/13 Aceito: 10/07/13 